\title{
La diversidad: el reto de la educación inclusiva
}

Dra. Bertha E. Martínez Ocaña

\section{RESUMEN}

En este artículo se revisan los conceptos diversidad, inclusión, inclusión educativa, destacando la diversidad como un valor que nos enriquece. Se presenta los acuerdos nacionales e internacionales que impulsan la educación inclusiva, como un derecho a la educación para Todos.

Palaras claves: Diversidad, Inclusión, Inclusión educativa.

\section{SUMMARY}

In this article we review the concepts of diversity, inclusion, educational inclusion, highlighting diversity as a value that enriches us. It presents the national and international agreements that promote inclusive education, as a right to education for All.

Keywords: Diversity, Inclusion, Educational inclusion.

La inclusión educativa en los últimos años viene ocupando un espacio privilegiado en las organizaciones de todos los países. Plantea nuevas formas de respuestas a la diversidad de las necesidades educativas en la escuela. Este tema es un reto educativo y social perviviendo en la actualidad. Es un planteamiento que requiere el trabajo de muchos sectores para abordar plenamente el problema.

UNESCO: "Lograr el derecho a la educación es el cimiento sobre el cual erigir una sociedad verdaderamente inclusiva en la que todos aprendan juntos y participen en un plano desigualdad. Sin embargo, en la actualidad, más de 77 millones de niños no asisten a la escuela y más de 781 millones de adultos no están alfabetizados. Esa exclusión se agudiza particularmente en el caso de las personas con discapacidad. Aproximadamente el $97 \%$ de los adultos con discapacidad no 
sabe leer ni escribir. Las estimaciones sobre el número de niños discapacitados que asisten a la escuela en los países en desarrollo oscilan entre menos del 1\% al 5\%. Los niños discapacitado representan más de un tercio de todos los niños que se encuentran fuera del sistema educativo (..) Sin embargo, es posible construir un mundo de inclusión con una legislación y unas políticas eficaces. Las campañas de sensibilización nos harán avanzar en esa dirección. (...).De modo que emprendamos las dos tareas: sensibilizar y formular políticas eficaces para un mundo de inclusión. Compartimos la responsabilidad de convertir esos sueños, esas visiones, ese compromiso en una realidad tangible". (Matzura, 2008, p.4).

La educación inclusiva en el sistema educativo significa un cambio de paradigma sobre la base de la percepción de los Derechos Humanos, que se enfoca en superar el modelo dela homogeneidad que durante décadas ha reproducido la exclusión de los involucrados por condiciones sociales, culturales, étnicas, lingüísticas, físicas y sociales.

El Perú adherido y comprometido con este nueva filosofía sostiene que la educación peruana se sustenta en el siguiente principio: "La inclusión, que incorpora a las personas con discapacidad, grupos sociales excluidos, marginados y vulnerables, especialmente en el ámbito rural, sin distinción de etnia, religión y sexo u otra causa de discriminación, contribuyendo así a la eliminación de la pobreza, la exclusión y las desigualdades". (Ley General de Educación, 2003, p.5).

En el Perú, la aplicación del enfoque de educación inclusiva de personas con discapacidad se inicia en el siglo pasado. Esto queda evidenciado en la Ley General de las Personas con Discapacidad 27050-1998. que estableció por primera vez en nuestro país, que ningún centro educativo podría negar la matrícula a una persona con discapacidad. Este enfoque será desarrollado por la Ley General de educación.

"La educación inclusiva es un proceso permanente, cuyo objetivo es ofrecer una educación de calidad para todos, respetando la diversidad y las distintas necesidades y aptitudes, características y expectativas de aprendizaje de los educandos y de las comunidades, eliminando toda forma de discriminación" (Unesco, 2013, p. 8) La desigualdad y la pobreza constituyen serios obstáculos para .lograr una educación plenamente inclusiva. Por esta razón, es imprescindible el desarrollo de políticas intersectoriales

Esta corriente, que surge a partir de la Conferencia Mundial de Educación para Todos, celebrada en 1990 en Jomtien (Tailandia) tiene por objetivo reestructurar las escuelas para poder responder a las necesidades de los niños. Lo que se sustenta en esta conferencia es quizá lo que de manera más decisiva y explícita ha contribuido a impulsar la Educación Inclusiva en todo el mundo. En la Convención de las Naciones Unidas sobre el Derecho de las Personas con Discapacidad, ratificada por España en 2008, se recoge el derecho a la educación inclusiva a todos los niveles así como la enseñanza a lo largo de la vida.

A partir de los acuerdos internacionales y nacionales, se ha modificado los conceptos, las políticas, las culturas y las prácticas educativas para promover la transformación del sistema escolar para atender a los estudiantes independientemente de sus características, necesidades y el contexto en que se desarrollen. 
El modelo de cambio que propician las escuelas inclusivas, constituyen un proceso de innovación educativa. No basta que los alumnos con necesidades educativas, estén en las escuelas, sino que deben participar en toda la vida escolar y la sociedad misma, no es lo mismo ubicarlos en una escuela que incluirlos.

El gran reto de la educación reside en ser capaz de ofrecer a cada estudiante la ayuda pedagógica que necesita, ajustar la intervención educativa a la individualidad del alumno.es decir adaptando la enseñanza a la capacidades, intereses y motivaciones de los estudiantes.

La educación inclusiva en el sistema educativo significa un cambio de paradigma sobre la base de la percepción de los Derechos Humanos, que se enfoca en superar el modelo dela homogeneidad que durante décadas ha reproducido la exclusión de los involucrados por condiciones sociales, culturales, étnicas, lingüísticas, físicas y sociales.

La educación inclusiva constituye un enfoque educativo basado en la valoración de la diversidad como un elemento que enriquece el proceso enseñanza aprendizaje, en consecuencia favorece el desarrollo humano. Parrilla (2003, p.98) "Hablar de diversidad nos remite a hablar de igualdad. La diversidad no es más que una manifestación de la igualdad inherente al género humano".

La educación inclusiva significa que todos los niños estudiantes de una determinada comunidad aprendan juntos, independientemente de sus condiciones sociales o culturales. incluidos aquellos que presentan discapacidad. Es una escuela que no pide requisitos de entrada para hacer efectivo los derechos a la educación y a la igualdad de oportunidades. (Parra Dussan, 2010).

Las ambiciones en el ámbito educativo se plasman de manera esencial en el Objetivo de Desarrollo Sostenible 4 que pretende garantizar una educación inclusiva y equitativa de calidad y promover oportunidades permanente de aprendizaje para todos (Educación 2030).

Unesco en el 2015, organizó el Foro Mundial sobre la Educación 2015 en Incheon (República de Corea) se debatió sobre el desarrollo avance de la Educación del 2000 al 2015. Al final de este foro se dio la Declaración de Incheon para la educación 2030, en la que presentan una nueva visión de la educación para los próximos quince años. Se sostiene que la Inclusión y la Equidad son la piedra angular de una agenda de la educación transformadora, y se comprometen hacer frente a todas las formas de exclusión y marginación. Asimismo se comprometen a realizar cambios necesarios en las políticas de educación y centrar las esfuerzos en los más desposeídos, especialmente en aquellos con discapacidad.

En el año 2017, la Declaración de Buenos Aires y los países de América Latina y el Caribe plasmó acuerdos sobre las prioridades educativas para el 2030. El documento regional indica que la educación debe contribuir a la eliminación de la pobreza, la reducción de las inequidades a través de una educación de calidad, inclusiva y el aprendizaje a lo largo de la vida"(Educación 2030, p.6).

Al respecto, Echeita (2006), un enfoque inclusivo modifica a todo el sistema y a toda la escuela. Ya no se trata que los estudiantes se adecúen a un currículo prescrito, sino que para ello el currículo 
tiene que ser más abierto, flexible adaptándose o diversificándose de acuerdo a las necesidades de los estudiantes. Por lo tanto esto va más allá de lo cognitivo, está referido a la motivación e intereses de los estudiantes.

Es decir, no basta con hacer modificaciones de planes, programas e infraestructuras, es necesario modificar nuestra manera de pensar, y considerar que todos somos diferentes y por lo tanto estas diferencias deben ser valoradas. Se trata de sensibilizar a la comunidad en general sobre la valiosa contribución que las personas con diversidad pueden hacer a ella, apoyando su participación activa y haciéndoles posible vivir una vida lo más satisfactoria posible.

En el año 2003, el gobierno del Perú estableció la Década de la Educación Inclusiva 2003- 2012, destinada a atender a los niños, jóvenes y personas con discapacidad: y destacando la necesidad de priorizar la educación inclusiva durante un período largo y garantizando su calidad y eficiencia. Señala el acceso y la atención temprana desde la primera infancia, con riesgo de discapacidad.

Tovar Sánchez (2013) refiere que en el Perú se han dado los primeros pasos para una adecuada inclusión de personas con discapacidad, pero los retos son grandes. Esto significa que la proporción de personas con discapacidad atendidas por el sistema educativo es muy pequeña, lo que lleva a una gran situación de exclusión. Así mismo los soportes pedagógicos no están garantizados, lo que compromete la calidad educativa. Sin embargo el derecho a la educación de las personas con discapacidad ha aparecido en la agenda política y social, las normas para su implementación muestran avances importantes.

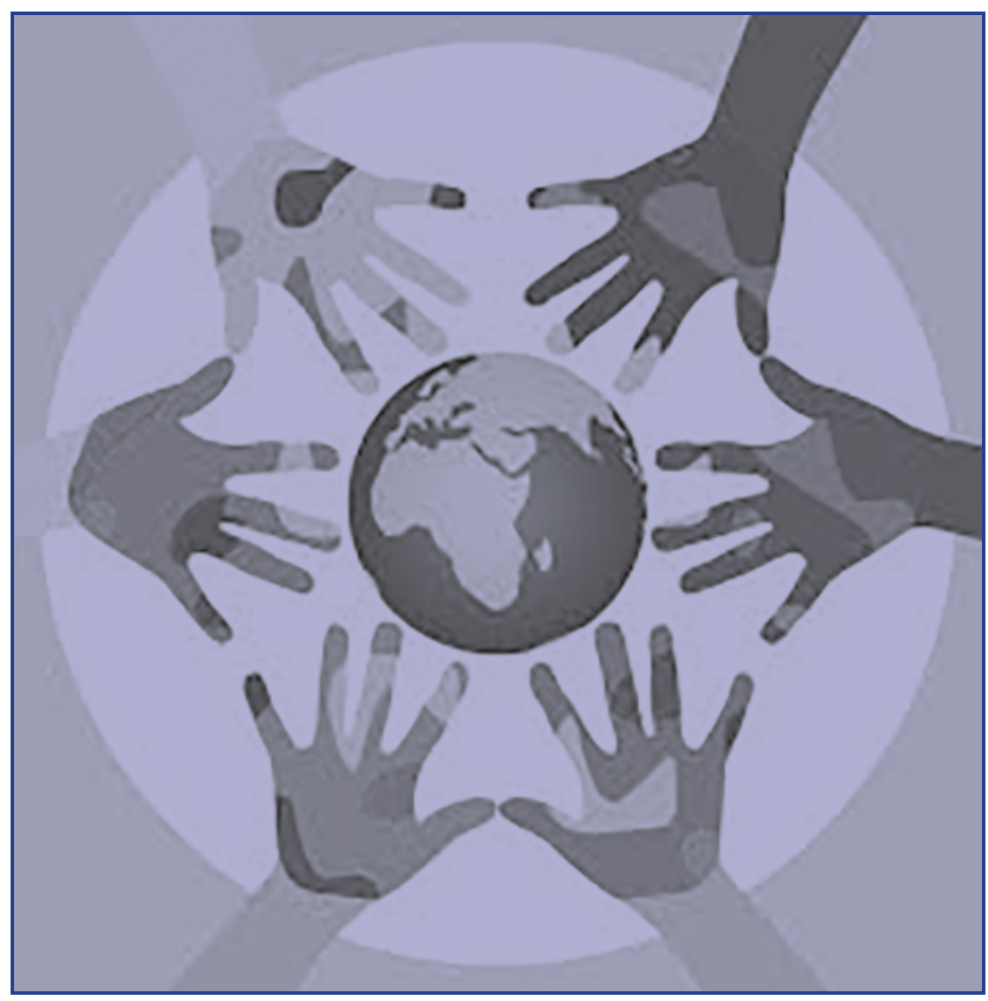




\section{ATENCIÓN A LA DIVERSIDAD}

El gran reto de la educación inclusiva es responder a la diversidad de los estudiantes. Pero no se trata solo de dar educación, sino que esta tiene que ser con calidad y equidad y esto no significa darle lo mismo a todos, sino darle lo que necesita.

Es indudable que la diversidad en todas sus manifestaciones, constituye un desafío para los sistemas educativos. Esto significa que las escuelas deben estar preparadas para acoger y educar a todos los estudiantes. Un programa de inclusión debe responder a las necesidades de los estudiantes y las escuelas deben ser un reflejo de su diversidad en la comunidad. Pero la inclusión es más que el acceso, implica la permanencia, participación y el desarrollo de sus potencialidades. Es decir, la inclusión se orienta hacia una educación de calidad sobre todo de aquellos que por distintas razones están excluidos o en riesgo de ser marginados, esto varía de un país a otro, pero generalmente son las personas con discapacidad.

Unesco: "Un proceso de fortalecimiento de la capacidad del sistema educativo para llegar a todos los educandos: por tanto, puede entenderse como una estrategia clave para alcanzar la educación para Todos (EPT). Como principio general, debería orientar todas las políticas y prácticas educativas, partiendo del hecho de que la educación es un derecho humano básico y el fundamento de una sociedad más justa y equitativa". (Citado por Parra Dussan 2010 p.10).

En este sentido la calidad de la educación desde el enfoque inclusivo, comprende un equilibrio entre excelencia y equidad. Es decir no se puede hablar de calidad cuando tan sólo una minoría de estudiantes aprende lo necesario para participar en la sociedad y desarrollar su proyecto de vida. La equidad significa proporcionar a cada persona las ayudas y los recursos que necesita para que esté en igualdad de condiciones de aprovechar las oportunidades educativas y de aprender a niveles de excelencia, con el fin de que la educación no reproduzca las desigualdades de origen de los estudiantes ni condicione sus opciones de futuro. (UNESCO, 2008). La personalización de las ayudas es un aspecto clave porque las políticas de equidad suelen caracterizarse por ofrecer lo mismo a todos, lo cual limita su potencialidad para promover el máximo desarrollo de las personas.

La equidad es un factor fundamental para conseguir un mayor desarrollo y una cultura de paz, basado en el respeto y valoración de las diferencias y en la tolerancia. Difícilmente se puede aprender a respetar las diferencias sino se convive con ellas, si las diferencias de cualquier tipo se obvian y se excluyen. (Blanco, 2008).

Una respuesta positiva a la diversidad del alumnado exige una educación en valores y una actuación en el ámbito de los recursos y de la política educativa que implica considerar la diversidad desde un punto de vista ético, respetar su carácter axiológico, darle un valor positivo, reconocer que es inherente a la naturaleza humana (Saénz, 1997). Exige cambios en las concepciones, actitudes, prácticas pedagógicas, a formación de docentes, y la organización de las escuelas.

Las instituciones tienen que revisar constantemente sus valores, organización, recursos, diseños curriculares y minimizar las barreras que presentan los estudiantes para participar y tener éxito en su aprendizaje, buscando las estrategias más adecuadas para dar respuesta a la diversidad de 
los estudiantes en sus estilos ritmos de aprendizaje, intereses, motivaciones etc., Se aspira que la escuela inclusiva construya espacios democráticos y participativos que faciliten el aprendizaje a convivir juntos en la diversidad.

Las políticas de equidad basadas en enfoques asistencialistas, compensatorios y focalizados han mostrado no ser las más adecuadas para lograr una mayor educación inclusiva y social. Es preciso avanzar hacia políticas de equidad que sitúen a las personas en el centro de un proceso de desarrollo humano sostenible, aumentando sus capacidades y opciones para vivir con dignidad, valorando la diversidad y respetando los derechos de todas las personas. (CEPAL, 2005).

\section{EL DOCENTE INCLUSIVO}

Una de las prioridades que plantea la diversidad es la referida al docente para hacer frente a las características heterogenias de los estudiantes, cuyas capacidades en situaciones sociales, son cada vez más diversas. El docente inclusivo es aquel que valora y se adapta a la diversidad del alumnado, consciente de que las diferencias en el aprendizaje son un recurso y un valor educativo.

La labor del docente debe estar orientada a: "Formar personas capaces de lograr su realización ética, intelectual, artística, cultural, afectiva, física, espiritual y religiosa; promoviendo la formación y consolidación de su identidad y autoestima y su integración adecuada y crítica a la sociedad, para el ejercicio de su ciudadanía en armonía con su entorno; así como el desarrollo de sus capacidades y habilidades para vincular su vida con el mundo del trabajo y para afrontar los incesantes cambios en la sociedad y el conocimiento". (Ley General de educación 2005,p.5).

La educación inclusiva asumida como un nuevo enfoque, exige que el trabajo pedagógico se centre en brindar apoyo a las necesidades educativas y al fortalecimiento de las cualidades de todo y cada uno de los estudiantes, para que se desarrollen seguros y con éxito.

En su labor docente debe apoyar a todos los alumnos. Considerar las opiniones diversas y llevar a cabo metodologías flexibles que se adapten a la heterogeneidad buscando más el proceso que el resultado. Asimismo debe promover estrategias de autorregulación de su aprendizaje (aprendizaje autónomo). Igualmente debe promover el aprendizaje cooperativo.

MINEDU (2010), el docente inclusivo es aquel que reconoce y valora las diferencias individuales y las concibe como una fuente de enriquecimiento personal y social. Es el que reivindica al centro educativo como el ambiente natural del niño o joven para desarrollarse y lograr aprendizajes en igualdad de condiciones y oportunidades. Promueve el cambio de actitudes y concepciones en la comunidad educativa para lograr un trabajo conjunto y participativo. Así mismo motiva a la innovación permanente de la institución educativa para mejorar su organización y su propuesta curricular.

La educación inclusiva supone, la implementación de parte del docente, de estrategias y recursos que ayuden a los estudiantes, escuela, maestros y padres de familia a enfrentar seguros el reto que implica hacer efectivo el derecho que tienen todos los niños, niñas jóvenes de acceder a la educación y beneficiarse de una enseñanza de calidad adecuada a las necesidades individuales 
de aprendizaje, ofreciendo una respuesta educativa diversificada, proponiendo una estructura curricular común a todos los alumnos.

El docente actúa como un mediador social que fomenta el respeto por el otro y la aceptación de la diversidad como aspecto inherente al ser. Atiende las necesidades de todos los estudiantes, realizando las adaptaciones necesarias para que todos desarrollen al máximo su potencia, debe saber que el aprendizaje académico y social es igual importante para todos. Igualmente debe tener presente que la comunidad escolar es un contexto social que afecta a la personalidad de todos los estudiantes.

Por todo lo mencionado se reconoce que la inclusión es un proceso, en el sentido que la escuela tiene que ir buscando maneras para responder a la diversidad. Para lograr esto se requiere un estilo de profesional en la que el profesorado practique actitudes y acciones positivas en su diario quehacer. Es necesario que los profesores reflexionen sobre sus prácticas inclusivas.

El tema de la diversidad es centro de interés de los profesionales de la educación, sin embargo no para todos la diversidad tiene el mismo significado, ni parte de los mismos supuestos ideológicos, ni presupone los mismos procesos de acción educativa (Jiménez, 1999).

Hay que destacar, que no es posible enseñar el respeto, la fraternidad, la tolerancia si no se propician modos de actuación en la escuela que favorezcan la práctica de estos valores. La tolerancia es un valor fundamental para comprender y promover la convivencia.

Por lo tanto, lograr una educación sensible a la diversidad de nuestros estudiantes, es contribuir al desarrollo de los individuos y las sociedades consolidando así los valores democráticos. Esta labor no involucra solo a la escuela, debe haber una colaboración estrecha entre escuela, familia y comunidad.

En efecto. "Por experiencia sabemos que es posible incluir a los estudiantes en las aulas siempre que los educadores hagan esfuerzos de acogerlos, fomentar amistades, adaptar el currículo y graduar las prácticas. No obstante la inclusión la inclusión plena no siempre se desarrolla con suavidad. En consecuencia, es vital que los adultos no opten por la vía fácil de excluir al niño, sino que busquen soluciones para lograr la inclusión social satisfactoria" (Stainback y Stainback, 1999. Citado por Parilla 2005, p.115).

No obstante, se puede decir que la educación inclusiva ha producido cambios en los docentes en cuanto a actitud, en sus evaluaciones y estrategias renovadas acordes con las necesidades educativas de los estudiantes.

Hoy en día el docente cuenta con el Servicio de Apoyo y Asesoramiento para la Atención de estudiantes con Necesidades Educativas Especiales SAANEE, es un recurso de apoyo para la inclusión está integrado por un equipo interdisciplinario conformado por profesionales docentes especializados en discapacidad intelectual, auditiva o visual, docentes con experiencia en autismo, docentes con experiencia en Educación Técnico Productiva, así como con profesionales no docentes y con experiencia en Educación Inclusiva, tales como psicólogos educacionales, psicólogos con 
experiencia en talento o superdotación, terapistas ocupacionales o de lenguaje, y trabajadores sociales.

SAANEE, funciona como una unidad operativa itinerante que tiene la responsabilidad de orientar y asesorar al personal directivo y docente de las instituciones educativas inclusivas de todos los niveles y modalidades del sistema educativo, para una mejor atención a los estudiantes (MINEDU,2012).

\section{BALANCE DE LA EDUCACIÓN INCLUSIVA}

Realizando el balance de la educación inclusiva en el Perú y teniendo como base los cuatro aspectos constitutivos del derecho a la educación ( disponibilidad, accesibilidad, aceptabilidad, adaptabilidad ) es evidente que existen avances limitados en cobertura y calidad. La explicación de lo limitado de los avances tienen que ver con el tema presupuestal, así como de la resistencia por parte de los actores en la gestión para la implementación de las políticas inclusivas, así como de la poca información de la sociedad. Siendo los derechos indivisibles e irrenunciables y no obstante de los limitados avances, no se justifican la crítica en contra de la política educativa inclusiva. (MINEDU, 2012).

No existe un seguimiento de los logros de aprendizaje después de aplicar las estrategias inclusivas ni se ha llevado a cabo una evaluación de la calidad educativa de las instituciones que han incluido a niños, niñas y jóvenes con discapacidad.

Los pasos o componentes para lograr una Educación Inclusiva en el Perú están presentes en la normatividad vigente, pero su secuencia lógica y su contenido no han sido suficientemente analizados y desarrollados.

La normatividad por sí sola no genera la transformación del sistema, se requiere del compromiso del sector con la niñez excluida por su condición de discapacidad, que debe ser la razón de las políticas educativas. Asimismo, es importante considerar una ruta para la construcción de escuelas inclusivas, que oriente el esfuerzo de sus actores.

\section{CONCLUSIONES}

Asumir la diversidad y valorar positivamente las diferencias nos conduce a un marco cultural, amplio y flexible en la que se respete y acepte la diversidad de las personas. Esta valoración influye en la forma de entender la respuesta educativa, que desde el centro educativo, se ofrece a la diversidad.

La educación inclusiva asumida como un nuevo enfoque, exige que el trabajo del docente se centre en brindar apoyo a las necesidades educativas y el fortalecimiento de las habilidades de todos y cada uno de los estudiantes para su desarrollo seguro y exitoso.

Lograr sistemas educativos más inclusivos requiere una firme voluntad de los involucrados que se refleje en el desarrollo de políticas educativas de largo plazo y que abarquen a diferentes sectores. 
Exige también marcos legales que establezcan derechos, responsabilidades y la provisión de los recursos necesarios.

No son nuestros sistemas educativos los que tienen derecho a ciertos tipos de niños. Es el sistema escolar el que hay que ajustar para satisfacer las necesidades de todos los niños.

B. Lindquist

\section{REFERENCIAS}

Blanco G.M. (2008). La Educación Inclusiva: El camino hacia el futuro. Una breve mirada a los temas de Educación Inclusiva. Aporte a las discusiones de los Talleres. Ginebra: Unesco.

Comisión Económica para América Latina y el Caribe. (2005). Panorama social de América Latina. Santiago de Chile, CEPAL.

Consejo Nacional de Educación (2015) .CNE Opina. Boletín del Consejo Nacional de Educación. No 39- Diciembre $2^{0} 15$.

Echeita, G. (2006). Del dicho al hecho hay un gran trecho. I. Congreso Internacional de Educación Especial. Concepción Chile. Chile.

Jiménez, P. y Vila, M. (1999). De la Educación Especial a la Educación en la Diversidad. Málaga: Algibe.

Ley General de Educación No 28044, 2003.Lima- Perú.

Mayo, L., Cueto, S. y Arregui, P.(2015). Políticas de Inclusión Educativa. Boletín del Consejo Nacional de Educación No 39. Diciembre 2015.

Ministerio de Educación (2010). Guía para Orientar la Intervención de los Servicios de Apoyo y Asesoramiento para la Atención de las Necesidades Educativas Especiales. Dirección General de Educación Básica Especial. Lima- Perú.

Ministerio de Educación (2012). Educación Básica Especial y Educación Inclusiva. Balances y Perspectivas. Dirección General de Educación Especial. Lima-Perú. 
Ministerio de Educación(s/a). Asumiendo un nuevo Rol. Módulo II- Sesión 2. Dirección General de Educación Básica Especial: Lima - Perú.

Ministerio de Educación. (2013) . Una Escuela de Calidad para todos. Dirección General de Educación Básica Especial. Lima- Perú.

Ministerio de Educación, Cultura y Deporte (2012). Educación Inclusiva. Iguales en la Diversidad. Clarificar nuestros Valores. Igualdad en la diversidad. Madrid: s/e.

Parra Dusan, C. (2010). Educación Inclusiva. Un Modelo de Educación para Todos. Revista ISEES No8.

Parrilla Latas, A. (2003). Acerca del origen y sentido de la Educación Inclusiva. Bilbao: Mensajero.

Sáenz, M.(1997). Educación Inclusiva para todos. México: Porrúa.

Tovar Sánchez, M.T. (2003). La década de la Educación Inclusiva 2003 - 20012.Para niños con discapacidad. Consejo Nacional de EADCAIÓN-Lima- Perú: AGL Gráfica color S.R.L.

UNESCO (2005) Guidelines for Inclusion. Ensuring Access to Education for All [Orientaciones para la inclusión. Asegurar el acceso a la Educación para Todos] Paris: Unesco.

UNESCO (2008). La educación inclusiva: El Camino hacia el Futuro. Ginebra: Unesco

UNESCO. (2008). Educación Inclusiva. Portal de Unesco: www.unesco.cl.

UNESCO. (2010). Temario Abierto sobre Educación Inclusiva. Santiago.

UNESCO. (2013). Inclusión EN LA Educación. Ginebra: Unesco.

UNESCO (2015). IX Y X Jornadas de Cooperación Educativa con Iberoamérica sobre Educación Especial e Inclusión. OREAL/Unesco. Santiago de Chile: Imbunche Ediciones Portales.

UNESCO. (2015). Educación 2030. Declaración de Incheon. Hacia una educación Inclusiva equitativa y un aprendizaje a lo largo de la vida para Todos. Incheon: Unesco.

UNESCO. (2017). Educación 2030. Declaración de Buenos Aires. Agenda Mundial de Educación 2030. 